\title{
Massive Pulmonary Embolism as a Complication of Coronary Angiography and Its Successful Treatment With Half-Dose Alteplase
}

\author{
Orcun Gurbuz ${ }^{\mathrm{a}, \mathrm{d}}$, Hakan Ozkan ${ }^{\mathrm{b}}$, Abdulkadir Ercan $^{\mathrm{a}}$, Gencehan Kumtepe ${ }^{\mathrm{a}}$, Ilker Hasan Karal ${ }^{\mathrm{c}}$
}

\begin{abstract}
A 64-year-old woman was admitted to our emergency room for shortness of breath, chest pain and syncope. Her symptoms started suddenly 2 hours before admission when she was resting at home. She had a history of coronary angiography 2 weeks ago and undergone surgical repair of femoral pseudoaneurysm. She was diagnosed with massive pulmonary embolism and successfully managed with halfdose alteplase and discharged with warfarin treatment 5 days after the admission. This case is presented in order to draw attention to this rare presentation and the use of half-dose systemic thrombolytic in a patient with a history of vascular surgery within 2 weeks.
\end{abstract}

Keywords: Complication of coronary angiography; Pulmonary embolism; Half-dose systemic thrombolytic therapy

\section{Introduction}

Invasive coronary angiography is still the gold standard test for identifying the presence and extent of coronary artery disease, despite promising improvement in non-invasive coronary imaging. As with any invasive procedure, it has well-known major and minor complications. Major complications such as death, emergency cardiac surgery, clinical myocardial infarction, and major bleeding or need for vascular surgery occur in less than $2 \%$ of the population [1]. The incidence of pseudoaneurysm after diagnostic angiography is reported between $0.5 \%$ and $2.0 \%$. Ultrasound-guided non-surgical treatment is currently

Manuscript accepted for publication August 27, 2014

aDepartment of Cardiovascular Surgery, School of Medicine, Balikesir University, 10010 Balikesir, Turkey

bepartment of Cardiology, Faculty of Medicine, Bahcesehir University, Istanbul, Turkey

'Department of Cardiovascular Surgery, Samsun Hospital for Education and Research, Ilkadim, 55090 Samsun, Turkey

${ }^{\mathrm{d} C}$ Corresponding Author: Orcun Gurbuz, Balikesir Universitesi Tip Fakultesi Kalp ve Damar Cerrahisi Anabilim Dali, Bigadic, 10010 Balikesir, Turkey. Email:gurbuzorcun@gmail.com

doi: http://dx.doi.org/10.14740/jmc1934w the first-line approach for iatrogenic femoral pseudoaneurysm unless it is too large [2]. Pulmonary embolism (PE) is rarely reported following coronary angiography $[3,4]$. The pressure applied to the groin or repair of access site complications after coronary angiography may cause PE. According to US Food and Drugs Administration (FDA), 2-h systemic recombinant tissue plasminogen activator (rt-PA) $100 \mathrm{mg}$ infusion is recommended for patients with massive acute PE [5]. However, systemic thrombolysis significantly increased major bleeding $[6,7]$. Therefore, several studies were conducted to assess the efficacy and safety of low-dose rt-PA in the treatment of acute $\mathrm{PE}$ and reported favorable results $[8,9]$.

\section{Case Report}

A 64-year-old woman was admitted to the emergency department for sudden onset of shortness of breath, chest pain and syncope. She had a coronary angiography in another hospital 2 weeks ago. Two days after her discharge, she had been rehospitalized to the same hospital due to swelling and pain in the right side groin area. Color Doppler ultrasound had identified a pseudoaneurysm of the right common femoral artery and surgical repair had been applied after the failure of ultrasonography-guided vascular compression therapy. Three days after her discharge she was admitted to the emergency department of our hospital with new-onset complaints of dyspnea, chest pain and syncope.

On physical examination, she presented a moderate general status, with a blood pressure $80 / 50 \mathrm{~mm} \mathrm{Hg}$, pulse 126 beats/ $\mathrm{min}$, respiratory rate 30 breaths $/ \mathrm{min}$, and an oxygen saturation $84 \%$. There were a $20 \times 15 \mathrm{~cm}$ ecchymosis and a $4 \mathrm{~cm}$ femoral surgical incision in her right groin. Laboratory test revealed a D-dimer level of $3,900 \mathrm{ng} / \mathrm{mL}$. The echocardiographic examination showed a large intracardiac thrombus with the expansion of the right heart chambers (Fig. 1, 2). Venous Doppler ultrasonography detected DVT in the left superficial femoral vein. Computed tomography of the chest revealed large thrombus in the main pulmonary artery.

A half-dose alteplase $(50 \mathrm{mg})$, in contrast to classical treatment, was administered intravenously within $2 \mathrm{~h}$. The patient's oxygen saturation and blood pressure came to the normal levels within approximately $90 \mathrm{~min}$ after the initiation of treatment. The control echocardiography on the next day revealed 


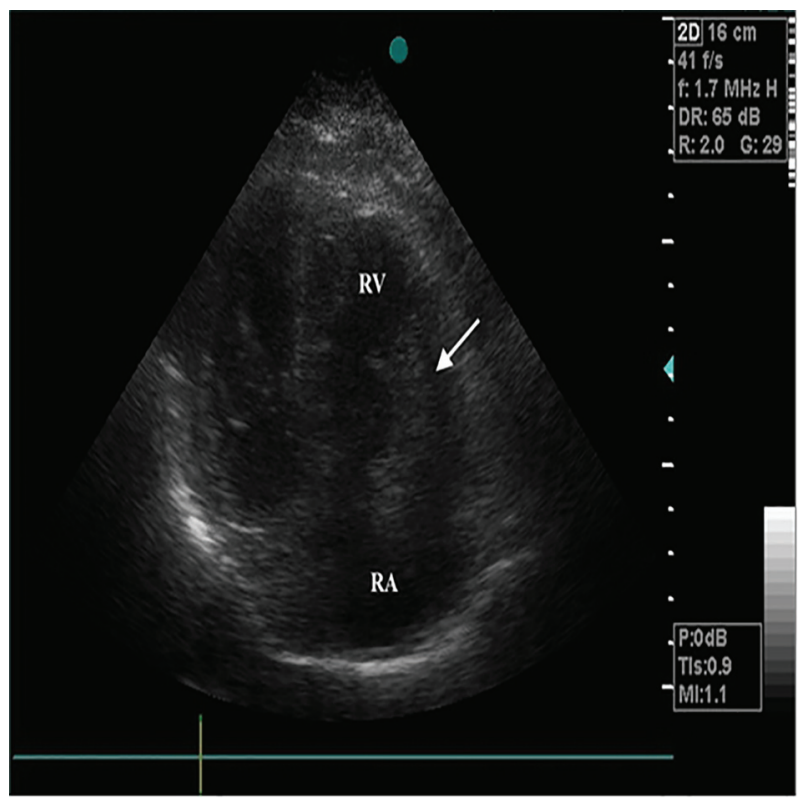

Figure 1. Apical four-chamber view showing the right atrial thrombus (arrow) and the enlargement of the right heart chambers. RA: right atrium; RV: right ventricle.

the complete disappearance of the intracardiac thrombus and right heart dilatation (Fig. 3). The patient was carefully monitored for evidence of bleeding. Low molecular weight heparin (LMWH) and warfarin therapy were started on the second day. LMWH was continued until the INR reached 2.5. The patient had no serious complication after half-dose systemic rt-PA and was discharged with warfarin treatment 5 days after the admission.

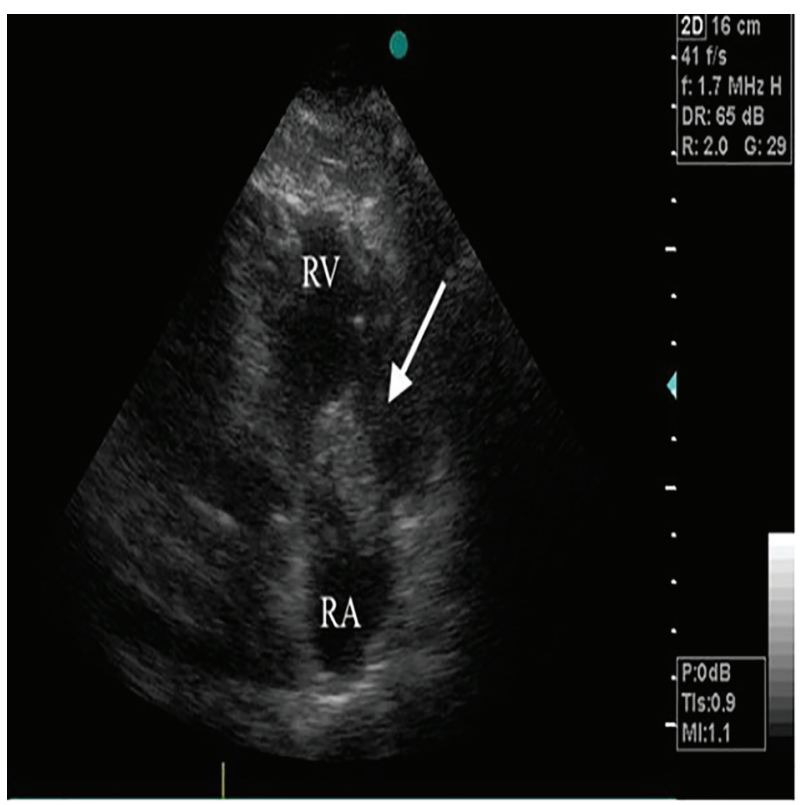

Figure 2. A modified four-chamber view showing atrial thrombus (arrow). RA: right atrium; RV: right ventricle.

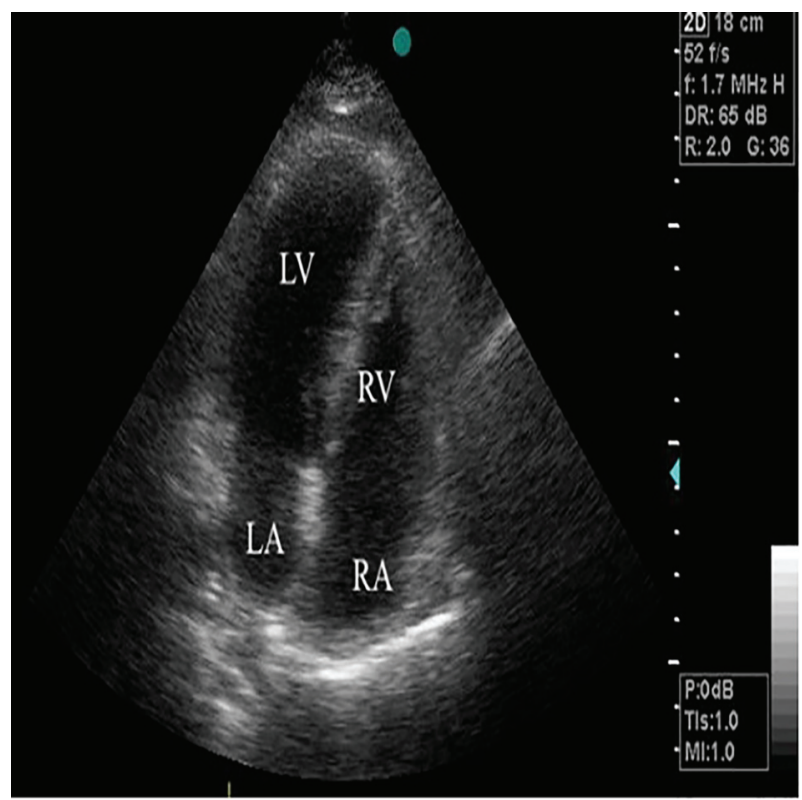

Figure 3. Transthoracic echocardiography (apical four-chamber view) $24 \mathrm{~h}$ after admission showing the normalization of right heart chambers and complete lysis of the right atrial thrombus. RA: right atrium; RV: right ventricle.

\section{Discussion}

As with any invasive procedure, coronary angiography has several complications such as death, emergency cardiac surgery, myocardial infarction, and major bleeding or need for surgical repair of vascular access [1].

The incidence of pseudoaneurysm after diagnostic angiography is reported between $0.5 \%$ and $2.0 \%$ [2]. Risk factors for the development of pseudoaneurysm include BMI $\geq 28 \mathrm{~kg} / \mathrm{m}^{2}$, larger number of cases performed per day in a particular room, larger sheath sizes, and preprocedural platelet count $<200 \times$ $10^{9} \mathrm{~L}[1]$. The main symptoms of femoral pseudoaneurysm are pain due to femoral nerve compression and extremity swelling secondary to venous compression which may also cause deep venous thrombosis.

Acute PE is often fatal, with a mortality rate of approximately $30 \%$ unless treated as soon as possible after symptoms develop [10]. PE is rarely reported following coronary angiography $[3,4]$. The pressure applied to the groin or access site complications such as hematoma or femoral pseudoaneurysm after coronary angiography may cause deep venous thrombosis which may lead to PE [3]. The recommended protocol for patients with massive acute PE or submassive PE with RV dysfunction is $100 \mathrm{mg}$ rt-PA given during a 2-h infusion [5]. Transcatheter procedures or emergency surgical thrombectomy are recommended as an alternative to thrombolysis when there are contraindications for thrombolysis or when thrombolysis has failed. Recent surgery, depending on the territory involved, is not necessarily barrier to fibrinolysis [7]. However, systemic thrombolysis may increase bleeding associated with surgery $[6,7]$. Recently, several studies were conducted to assess the 
efficacy and safety of low-dose rt-PA in the treatment of acute $\mathrm{PE}$ and reported similar efficacy but lower bleeding than standard dose of rt-PA $[8,9]$.

Considering patient clinical situation and for decreasing bleeding risk, we decided to administer $50 \mathrm{mg}$ rt-PA. The thrombus in right heart chamber was resolved completely within a day and the patient's health condition is back to normal within a day.

In conclusion, we assume that the cause of PE in our patient was transient deep venous thrombosis due to inguinal hematoma or femoral pseudoaneurysm. Optimal placement of the arterial sheath, use of smaller sheaths, and careful manual compression with shorter period of immobilization, can significantly reduce the incidence of deep venous thrombosis and PE. Moreover, half-dose rt-PA may be safe and effective treatment alternative in patients with massive PE having high bleeding risk with systemic thrombolysis.

\section{Competing Interests}

The authors declare that they have no competing interests.

\section{Financial Disclosures}

This manuscript did not receive any financial aid. The authors have no disclosures regarding this manuscript.

\section{Author Contributions}

Orcun Gurbuz and Gencehan Kumtepe planned the study and the structure of the manuscript. Abdulkadir Ercan did the final English writing of the text. Hakan Ozkan participated and contributed to the evaluation of the case as the cardiologist. Ilker Hasan Karal aided in the collection of data from the literature.

\section{References}

1. Tavakol M, Ashraf S, Brener SJ. Risks and complications of coronary angiography: a comprehensive review. Glob J Health Sci. 2012;4(1):65-93.
2. Stone PA, Campbell JE. Complications related to femoral artery access for transcatheter procedures. Vasc Endovascular Surg. 2012;46(8):617-623.

3. Shammas RL, Reeves WC, Mehta PM. Deep venous thrombosis and pulmonary embolism following cardiac catheterization. Cathet Cardiovasc Diagn. 1993;30(3):223-226.

4. Kivrak T, Durmus E, Atas H, Sunbul M, Sari I. A Rare Case of Acute Pulmonary Embolism after Coronary Angiography due to Sand Bag Compression. Int J Angiol. 2013;22(4):255-258.

5. Alonso-Coello P, Bellmunt S, McGorrian C, Anand SS, Guzman R, Criqui MH, Akl EA, et al. Antithrombotic therapy in peripheral artery disease: Antithrombotic Therapy and Prevention of Thrombosis, 9th ed: American College of Chest Physicians Evidence-Based Clinical Practice Guidelines. Chest. 2012;141(2 Suppl):e669S-690S.

6. Kearon C, Akl EA, Comerota AJ, Prandoni P, Bounameaux H, Goldhaber SZ, Nelson ME, et al. Antithrombotic therapy for VTE disease: Antithrombotic Therapy and Prevention of Thrombosis, 9th ed: American College of Chest Physicians Evidence-Based Clinical Practice Guidelines. Chest. 2012;141(2 Suppl):e419S-494S.

7. Jaff MR, McMurtry MS, Archer SL, Cushman M, Goldenberg N, Goldhaber SZ, Jenkins JS, et al. Management of massive and submassive pulmonary embolism, iliofemoral deep vein thrombosis, and chronic thromboembolic pulmonary hypertension: a scientific statement from the American Heart Association. Circulation. 2011;123(16):1788-1830.

8. Canan Hasanoglu H, Hezer H, Karalezli A, Arguder E, Kilic H, Senturk A, Er M, et al. Half-dose recombinant tissue plasminogen activator treatment in venous thromboembolism. J Investig Med. 2014;62(1):71-77.

9. Zhang Z, Zhai ZG, Liang LR, Liu FF, Yang YH, Wang C. Lower dosage of recombinant tissue-type plasminogen activator (rt-PA) in the treatment of acute pulmonary embolism: a systematic review and meta-analysis. Thromb Res. 2014;133(3):357-363.

10. Horlander KT, Mannino DM, Leeper KV. Pulmonary embolism mortality in the United States, 1979-1998: an analysis using multiple-cause mortality data. Arch Intern Med. 2003;163(14):1711-1717. 